\title{
Inequalities in outcomes for non-small cell lung cancer: the influence of clinical characteristics and features of the local lung cancer service
}

\author{
Anna L Rich, ${ }^{1}$ Laila J Tata, ${ }^{1}$ Catherine M Free, ${ }^{2}$ Rosamund A Stanley, ${ }^{3}$ \\ Michael D Peake, ${ }^{2}$ David R Baldwin, ${ }^{4}$ Richard B Hubbard ${ }^{1,5}$
}

\begin{abstract}
See Editorial, p 1023
${ }^{1}$ Division of Epidemiology and Public Health, University of Nottingham, Nottingham, UK ${ }^{2}$ Department of Respiratory Medicine, University Hospitals of Leicester, Glenfield Hospital Leicester, UK

${ }^{3}$ Audit Support Unit, The NHS Information Centre for Health and Social Care, Leeds, UK ${ }^{4}$ Department of Respiratory Medicine, Nottingham University Hospitals, Nottingham, UK

${ }^{5}$ Respiratory Medicine Biomedical Research Unit, Nottingham City Hospital, Nottingham, UK
\end{abstract}

\section{Correspondence to} Dr Anna L Rich, Clinical Sciences Building, City Campus, Hucknall Road, Nottingham NH5 1PB, UK:

anna.rich@nottingham.ac.uk

Received 6 January 2011 Accepted 23 June 2011 Published Online First 23 July 2011

\section{ABSTRACT}

Background The treatment given to patients with lung cancer and survival vary between and within countries. The National Lung Cancer Audit (NLCA) linked to Hospital Episode Statistics was used to quantify the extent to which these outcomes are influenced by patient features and/or hospital facilities and performance indicators.

Methods All patients with a histological diagnosis of non-small cell lung cancer (NSCLC) were included.

Logistic regression was used to quantify the independent influence of features of both patients and hospitals on the likelihood of having surgery and Cox regression was used for survival analyses.

Results There were 34513 patients with NSCLC in our dataset. After adjusting for age, sex, performance status, stage and Charlson Index of comorbidity, patients with NSCLC first seen in thoracic surgical centres $127 \%$ of the cohort) were $51 \%$ more likely to have surgery than those seen in non-surgical centres ladjusted OR 1.51, 95\% Cl 1.16 to 1.97$)$. Resection rates varied from $13 \%$ to $17 \%$ between non-surgical and thoracic surgical centres. Surgery was the most powerful determinant of overall survival (adjusted HR 0.41, 95\% Cl 0.39 to 0.44).

Conclusion A minority of patients with NSCLC first seen in a thoracic surgical centre are more likely to have surgery and to benefit from the survival advantage this confers. This finding suggests that there is an opportunity to improve the outcome for patients with lung cancer in England by optimising access to thoracic surgeons in non-surgical centres.

\section{INTRODUCTION}

Lung cancer is the commonest cause of cancerrelated death worldwide. Non-small cell lung cancer (NSCLC) accounts for more than $80 \%$ of all lung cancers ${ }^{1} 2$ and, for these people, surgical resection represents the best chance of cure. ${ }^{3}$ The National Institute for Health and Clinical Excellence (NICE) recommended surgical resection for all patients with stage I and II disease who had no medical contraindications and adequate lung function. ${ }^{4}$ At present only a small minority of people with NSCLC will have disease which is suitable for surgical resection..$^{5}$ There is evidence that surgical resection rates for lung cancer and survival from the disease vary between and within countries. ${ }^{2}$ 6-8 This variation is anecdotally attributed to geographical differences in patient features-for example, individuals from one area being older,

\section{Key messages}

What is the key question?

- Is the variation in surgical resection rates across England primarily due to patient features or to the features of the NHS trust where a patient is first seen?

\section{What is the bottom line?}

- Having adjusted for all patient features (including co-morbidity), patients with NSCLC who are first seen in a thoracic surgical centre are $50 \%$ more likely to have surgery than those first seen in a non-surgical centre.

\section{Why read on?}

- Findings suggest that changes to the patient pathway could increase the number of people who are offered potentially curative surgery.

presenting with late stage disease or having significant comorbidities which preclude an anaesthetic. Our intention was to adjust for all patient features and investigate whether the variation in outcomes at a hospital level were still evident.

In this study we have used the National Lung Cancer Audit (NLCA) dataset linked to Hospital Episode Statistics to quantify the influence of patient and NHS trust level features on access to surgery and survival for people with NSCLC in England. The NHS trust is the hospital where an individual is first seen, and 'trust level features' is the term used in this paper to describe markers of performance and the facilities available there. There are 157 NHS trusts in England and only 31 have thoracic surgery available on-site. There are 28 cancer networks in England. These networks cover a geographical area and bring together all those responsible for the commissioning and delivery of cancer care. Within each one there is a 'hub and spoke' system whereby specialist cancer centres, with thoracic surgeons available on-site, act as the 'hub' and provide specialist cancer services such as surgery and radiotherapy for the hospitals that compose the 'spokes'. Some thoracic centres are purely thoracic, others are cardiothoracic, but we have used the term 'thoracic surgical centre' as a means of describing the service they provide regardless of the subspecialty of the surgeons. 


\section{METHODS}

Data for all English patients first seen between January 2004 and 31 December 2008 were downloaded from the NLCA for us by the NHS Information Centre for Health and Social Care. For this study we restricted our analyses to patients with a proven histological diagnosis of NSCLC. Our initial dataset included information on histological subtype, sex, age at diagnosis, socioeconomic status (census derived Lower Super Output Area Townsend Score), performance status (as classified by the Eastern Cooperative Oncology Group), stage at presentation (as classified by the American Joint Committee on Cancer and Union Internationale Contre le Cancer version 6), details of the NHS trust where a patient was first seen and whether surgical resection had occurred.

Although the NLCA records information on comorbid illness, these data are incomplete and are limited to only six disease groups. The audit records only whether or not the presence of this comorbid illness influenced the treatment decision. We therefore obtained permission to link the NLCA to Hospital Episode Statistics to provide information on inpatient episodes and diagnoses. The Hospital Episode Statistics dataset contains up to 20 diagnoses for each hospital episode coded using ICD-10, and our linked dataset covered the 11 financial years between 1997 and 2008. We used these data to calculate a Charlson Index, ${ }^{9}$ which is a composite score of comorbidity which has been validated in cohorts of men and women with both malignant and non-malignant diseases. We excluded the ICD-10 codes for lung cancer in our calculations. To minimise bias resulting from reverse causation, we ignored the last 3 months of Hospital Episode Statistics data prior to the date of lung cancer diagnosis. This time period was arbitrarily chosen by the authors to avoid the bias that would arise if a patient appeared to develop a new comorbid disease shortly before the diagnosis of lung cancer was made and the two conditions were linked. We then divided the cohort into four groups on the basis of their Charlson score. We also used data from the Hospital Episode Statistics dataset to provide information on ethnicity.

In order to describe variation in the facilities available at NHS trusts, we created three binary variables: (1) whether or not a trust is a thoracic surgical centre; (2) whether or not it is a radiotherapy centre; and (3) whether or not the centre was actively entering patients into clinical trials for lung cancer. We defined a centre as being actively involved with lung cancer clinical trials if they were in the top $30 \%$ of trusts in terms of the proportion of their patients that were entered into clinical trials. To calculate these figures we used information supplied by the National Cancer Research Network on the number of trial participants for each NHS trust and our previous estimates of the total number of patients with lung cancer seen at each NHS trust. ${ }^{10}$ A fourth binary variable thought to quantify the performance of the lung cancer multidisciplinary team was also created based on the results of the peer review process 2004-7. We took the overall score for each NHS trust and defined as 'excellent' any NHS trust which was in the top quartile. However, this was subsequently dropped from multivariate regression analyses due to the lack of evidence to support the assumption that it was influential.

We performed logistic regression analyses to estimate the ORs for receiving surgery, adjusting our analyses for all patient and NHS trust level features and clustering on NHS trusts. In order to look more specifically at variation in access to surgery in patients in whom we would expect to have a high chance of undergoing surgery, we repeated our analyses in a subgroup of patients with stage I or II disease.
For our survival analyses we used the date of diagnosis recorded in the NLCA as our start date where available and, in the absence of this, we interpolated a surrogate date of diagnosis using the date of first clinic appointment and the median interval between these dates for the cohort overall (10 days). The end date was either the date of death or the date of last data collection (30 September 2009). Because some patients with lung cancer are diagnosed at postmortem examination, we excluded people from the survival analysis when the date of death was the same as or earlier than the date of diagnosis. Cox regression analyses were used to calculate HRs and a multivariate model was constructed to mutually adjust for all patient and NHS trust features. The final Cox regression model included clustering by NHS trusts. We then repeated our survival analysis for the subgroup of patients with stage I and II disease as outlined above. We checked the proportional hazards assumption for our model by inspecting Nelson-Aaleen plots.

Finally, to determine whether people first seen at a thoracic surgical centre were different from those seen at a non-surgical centre, the demographic features of the two patient groups were compared and, for the subgroup of patients who had undergone surgery, we estimated survival according to where the patient was first seen.

\section{RESULTS}

Our NLCA dataset contained a total of 87252 patients who were first seen at an English NHS trust between January 2004 and 31 December 2008. Histological diagnosis was based on a pretreatment histology examination as this would be the information available to multidisciplinary teams. Data in the early years of the NLCA were poorly completed and so 33964 (42\%) of this dataset had no pretreatment histology recorded. Small cell lung cancer accounted for 7845 patients (10\%), 2772 (3\%) had mesothelioma and $1872(2 \%)$ had other diagnoses. There were 34513 patients (43\%) with a histological diagnosis of NSCLC; $33 \%$ of patients had squamous cell carcinoma, $27 \%$ had adenocarcinoma, $33 \%$ had NSCLC not otherwise specified and the remaining $7 \%$ had large cell tumours, bronchoalveolar cell carcinoma and carcinoma-in-situ. The median age at diagnosis was 71 years (IOR 63-77) and 60\% were male. A total of 4485 patients $(13 \%)$ underwent a surgical procedure. The median Charlson Index at diagnosis was 1 (IOR 0-2), with a minimum score of 0 and a maximum score of 17 . There are 31 cardiothoracic surgical centres in England, 49 radiotherapy centres and 44 NHS trusts which were defined as trial active, which was equivalent to putting $5 \%$ of more of their patients with lung cancer into clinical trials. Among the thoracic surgical centres, 19 were also radiotherapy centres and 15 were trial-active. Twelve NHS trusts possessed all three features. Of the 34513 patients, $9168(27 \%)$ were first seen in thoracic surgical centres.

\section{Access to surgery}

Table 1 shows the results of univariate and multivariate logistic regression analyses for the associations between having surgery and patient and NHS trust features. As the Charlson Index increased, indicating a higher level of comorbid illness, the likelihood of having surgery decreased. The OR for having surgery, mutually adjusted for all patient and NHS trust features, for patients with a Charlson Index of $\geq 4$ compared with patients with a Charlson index of 0 was 0.67 (95\% CI 0.56 to 0.80 ). Patients in the fourth age quintile (76-80 years) were almost half as likely to undergo surgery as those in the youngest quintile (adjusted OR $0.56,95 \%$ CI 0.49 to 0.56 ). Patients with 
Table 1 Result of logistic regression analyses evaluating the influence of patient and NHS trust level features on the likelihood of receiving surgery clustered by NHS trust $(n=34513)$

\begin{tabular}{|c|c|c|c|c|c|c|}
\hline & No of patients & $\begin{array}{l}\text { No who received } \\
\text { surgery }\end{array}$ & $(\%)^{*}$ & Unadjusted OR (95\% CI) & Adjusted OR† (95\% Cl) & p Value \\
\hline \multicolumn{7}{|l|}{ Sex } \\
\hline Male & 20945 & 2637 & 13 & & & \\
\hline \multicolumn{7}{|l|}{ Age quintile } \\
\hline 1 (30-62 years) & 8221 & 1290 & 16 & & & $<0.001 \neq$ \\
\hline 2 (63-69 years) & 7635 & 1232 & 16 & $1.03(0.95$ to 1.13$)$ & $0.95(0.85$ to 1.05$)$ & \\
\hline 5 (81-100 years) & 4849 & 261 & 5 & $0.31(0.27$ to 0.35$)$ & $0.26(0.22$ to 0.32$)$ & \\
\hline \multicolumn{7}{|l|}{ Stage } \\
\hline IA & 1178 & 722 & 61 & & & $0.001 \neq$ \\
\hline IB & 2138 & 1029 & 48 & 0.59 (0.51 to 0.68$)$ & $0.63(0.52$ to 0.76$)$ & \\
\hline$\| A$ & 202 & 121 & 60 & $0.94(0.70$ to 1.28$)$ & $0.96(0.72$ to 1.28$)$ & \\
\hline IIB & 1448 & 515 & 35 & $0.35(0.30$ to 0.41$)$ & $0.35(0.28$ to 0.43$)$ & \\
\hline Missing & 10325 & 1135 & 11 & $0.08(0.07$ to 0.09$)$ & $0.09(0.07$ to 0.12$)$ & \\
\hline \multicolumn{7}{|l|}{ Performance status } \\
\hline 0 & 5847 & 1651 & 28 & & & $<0.001 \neq$ \\
\hline 1 & 9282 & 1372 & 15 & $0.44(0.41$ to 0.48$)$ & $0.52(0.46$ to 0.59$)$ & \\
\hline 2 & 5317 & 264 & 5 & $0.13(0.12$ to 0.15$)$ & $0.19(0.15$ to 0.24$)$ & \\
\hline 3 & 3251 & 67 & 2 & $0.05(0.04$ to 0.08$)$ & $0.10(0.08$ to 0.14$)$ & \\
\hline 4 & 760 & 13 & 2 & $0.04(0.03$ to 0.88$)$ & $0.11(0.06$ to 0.18$)$ & \\
\hline Missing & 10056 & 1118 & 11 & $0.32(0.29$ to 0.35$)$ & $0.40(0.32$ to 0.49$)$ & \\
\hline \multicolumn{7}{|l|}{ Ethnic group } \\
\hline White & 26511 & 3443 & 13 & & & $0.39 \S$ \\
\hline Black & 234 & 27 & 12 & $0.87(0.58$ to 1.31$)$ & 0.84 (0.47 to 1.49$)$ & \\
\hline 4 & 7148 & 942 & 13 & 0.97 (0.87 to 1.08$)$ & 0.98 (0.84 to 1.13$)$ & \\
\hline 5 (least affluent) & 9051 & 1110 & 12 & 0.89 (0.81 to 0.99$)$ & 0.86 (0.71 to 1.04$)$ & \\
\hline Missing & 77 & 4 & 5 & $0.35(0.13$ to 0.96$)$ & $0.43(0.15$ to 1.23$)$ & \\
\hline \multicolumn{7}{|l|}{ Charlson Index } \\
\hline 0 & 15573 & 2341 & 15 & & & $<0.001 \neq$ \\
\hline 1 & 6951 & 985 & 14 & $0.93(0.86$ to 1.01$)$ & 0.95 (0.86 to 1.04$)$ & \\
\hline 2 or 3 & 5828 & 752 & 13 & $0.83(0.77$ to 0.91$)$ & $0.89(0.80$ to 0.99$)$ & \\
\hline $4+$ & 6161 & 407 & 7 & $0.40(0.36$ to 0.45$)$ & $0.67(0.56$ to 0.80$)$ & \\
\hline \multicolumn{7}{|l|}{ Surgery centre } \\
\hline No & 25248 & 2947 & 12 & & & \\
\hline Yes & 9265 & 1538 & 17 & 1.51 (1.41 to 1.61$)$ & 1.51 (1.16 to 1.97$)$ & $<0.001$ \\
\hline \multicolumn{7}{|l|}{ Radiotherapy centre } \\
\hline No & 21646 & 2614 & 12 & & & \\
\hline Yes & 12867 & 1871 & 15 & 1.24 (1.16 to 1.32$)$ & $1.02(0.83$ to 1.27$)$ & 0.854 \\
\hline \multicolumn{7}{|l|}{ Trial entry } \\
\hline 'Poor' & 23136 & 2817 & 12 & & & \\
\hline 'Good' & 11377 & 1668 & 15 & 1.24 (1.16 to 0.32$)$ & $1.15(0.88$ to 1.50$)$ & 0.34 \\
\hline
\end{tabular}

*Percentage of each variable who received surgery.

tOR for surgery adjusted for all other variables in the table.

$\neq p$ for trend.

$\S$ Result of a log likelihood ratio test.

more advanced disease stage and poorer performance status were also less likely to have surgery. There was no evidence that ethnic group or socioeconomic status influenced the likelihood of having surgery.
If a patient was first seen in a thoracic surgical centre they were $51 \%$ more likely to have surgery, even after adjusting for all of the patient level features (adjusted OR 1.51, 95\% CI 1.16 to 1.97). There was some evidence that this difference between 
surgical and non-surgical centres varied year on year (test for interaction, $\mathrm{p}=0.003$ ), but there was no obvious trend over time as the ORs for 2004, 2005, 2006, 2007 and 2008 were 1.58 (95\% CI 0.82 to 3.05$), 2.50$ (95\% CI 1.57 to 3.88$), 1.80$ (95\% CI 1.18 to 2.72 ), 1.11 ( $95 \%$ CI 0.76 to 1.64 ) and 1.47 (95\% CI 1.09 to 1.99 ), respectively. In our multivariate analyses, whether the NHS trust was a radiotherapy centre or an active trial centre did not influence the likelihood of having surgery. The overall score at peer review had no influence on the likelihood of receiving surgery. Of those individuals first seen in an 'excellent' NHS trust, $943(12 \%)$ had surgery compared with those first seen in a 'standard' NHS trust (adjusted OR 0.97 (95\% CI 0.88 to 1.06)). We identified 4966 patients who had stage I or II disease, 2387 of whom (48\%) had surgery. In these patients the likelihood of having surgery was $53 \%$ higher in patients first seen at NHS trusts that were thoracic surgical centres than in those seen in non-surgical centres (adjusted OR 1.53, 95\% CI 1.09 to 2.13).

\section{Survival analysis}

A small number of patients $(n=148)$ had a date of death on or before the date of diagnosis and so were excluded from the survival analyses. In our survival analyses women had a better prognosis than men but, as age, stage and performance status increased, the prognosis worsened (table 2). Patients with a Charlson Index score of $\geq 4$ had an adjusted HR of 1.59 (95\% CI 1.52 to 1.66) compared with those with a Charlson Index score of 0 . There was no evidence that social deprivation was linked to worse survival. Whether the NHS trust where a patient was first seen was a thoracic surgical centre or not had no significant effect on overall mortality. The results of peer review had no effect on overall mortality (adjusted HR 1.01, $95 \%$ CI 0.98 to 1.04 ). There was no evidence that our proportional hazards assumption was incorrect.

Patients who had surgery had an almost $60 \%$ lower overall mortality (adjusted HR $0.41,95 \%$ CI 0.39 to 0.44 ) compared with those who did not have surgery, even after adjusting for all patient features. In the subgroup of people with stage I or II disease where $48 \%$ had surgery, the fully adjusted HR was very similar at 0.41 (95\% CI 0.37 to 0.46 ). The median survival for patients with stage I or II disease who had surgery was 774 days (IOR 305-2150) compared with a median survival of only 174 days (IOR 63-394) for those who did not have surgery.

The demographic features of patients first seen at thoracic surgical and non-surgical centres were similar (table 3), although the proportion of patients from the least affluent quintile of society was higher for the thoracic surgical centres than the nonsurgical centres $(p<0.001)$. Of the $4485(13 \%)$ patients who had surgery, $34 \%$ were first seen in a thoracic surgical centre. Survival after surgery did not appear to be related to where the patient was first seen (adjusted HR 1.01, 95\% CI 0.87 to 1.19).

\section{DISCUSSION}

Our results demonstrate that the likelihood of having surgery for patients with a histological diagnosis of NSCLC is independently influenced by patient level features including age, stage, performance status and comorbidity, and also by whether or not the patient is first seen at a thoracic surgical centre. Even after allowing for patient level features, those first seen at a surgical centre were $51 \%$ more likely to have an operation. This difference persisted among the subgroup of patients with early stage disease where surgery would be the preferred treatment modality and one might expect little variation in practice.

Our results also show that female sex, younger age, good performance status at diagnosis and early stage disease were all associated with better survival. By linking in information from Hospital Episode Statistics, we were also able to quantify comorbid illness relating to hospital admissions by calculating a Charlson Index and found that, as this score increased, survival became poorer. Whether or not an individual had surgery as part of their treatment plan was also an important determinant of survival, with those who did have surgery having a $60 \%$ reduction in their likelihood of death. This difference was identical in our subgroup of patients with early stage disease. In order to determine whether the higher surgical resection rates for patients first seen at thoracic surgical centres reflected these centres operating on patients with more advanced disease and/or a worse prognosis, we compared the patient features between people seen first at a thoracic surgical centre and those seen elsewhere. We found no difference in either survival or patient level features, with the exception that the thoracic surgical centres had a higher proportion of patients from more deprived backgrounds. This suggests that, if the clinical pathway was altered to ensure that the $73 \%$ of patients first seen at a nonsurgical centre had the same chance of having surgery as those first seen at a thoracic surgical centre, this would increase the overall resection rate in this patient group from 13\% to $17 \%$ with no detrimental impact on survival after surgery.

Although the NLCA is not mandatory, there is evidence within their annual reports that case ascertainment has increased steadily and is now in excess of $90 \% .{ }^{11-13}$ We have previously studied the validity of this dataset and have found no evidence of bias dependent on the levels of reporting by individual NHS trusts, ${ }^{10}$ which provides reassurance that the dataset reflects the full spectrum of lung cancer in England. Although a large proportion of our cohort had missing data for histology, we were still able to analyse a subgroup of more than 30000 patients with histologically-proven NSCLC. Alongside case ascertainment, data completeness has improved year on year. ${ }^{11} 12$ Our marker of comorbid illness was derived from codes relating only to hospital admissions and so will not have captured details of conditions managed independently by general practitioners. This means that, despite being a strong predictor of both survival and having surgery, our calculated Charlson Indices may be too low and this raises the possibility of residual confounding by comorbidity. However, when we adjusted our model for surgical resection and site first seen, the OR for Charlson Index did not change at all, suggesting that comorbidity is not a confounder for this association. The distribution of Charlson Indices in this cohort is very similar to those in both general practitioner datasets ${ }^{14}$ and cohorts of patients with NSCLC. ${ }^{15} 16$ One potential weakness is that there is no information on whether some patients who were offered surgery declined this intervention. Our research does not represent a randomised controlled trial looking at the impact of surgery in patients with NSCLC, and there has never been such a trial. The National Lung Cancer Screening Trial (USA) was recently stopped because the primary outcome of a significant reduction in mortality from lung cancer was reached (>20\%). This study compared chest x-ray with CT scanning and patients found to have lung cancer at a sufficiently early stage were treated surgically. This is the first time that screening has been shown to reduce mortality. ${ }^{17}$ What we are able to report are observational data from a large unselected cohort which illustrate the survival advantage of surgery in spite of adjusting for many patient features. It is possible that our results are still subject to some residual confounding or selection bias and this may mean that the marked benefit of surgery we have observed may be an overestimate of the true benefit. 
Table 2 Results of Cox regression analyses using all patient features, all NHS trust level features, surgical intervention or not and clustering by NHS trust $(n=34365)$

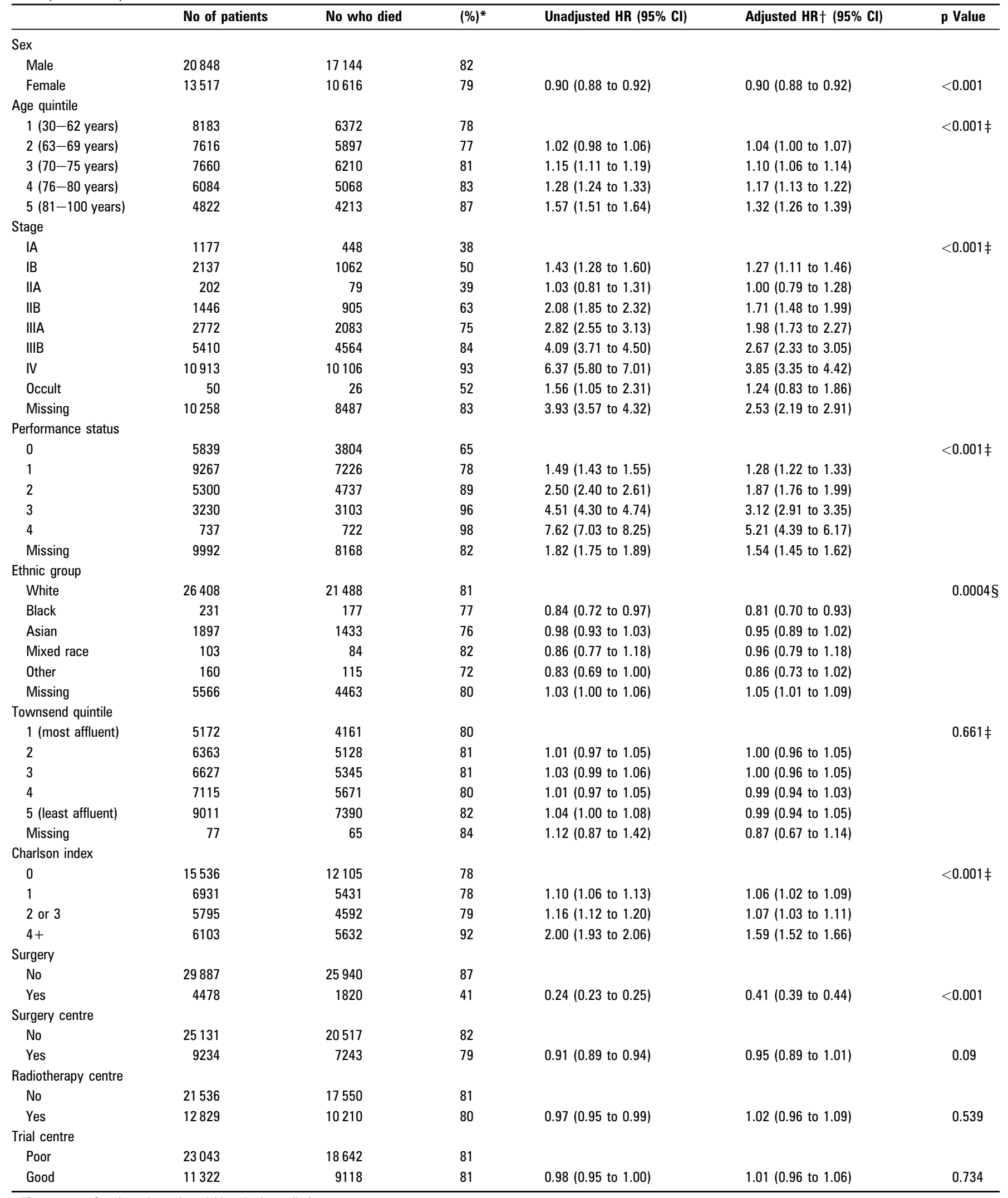

*Percentage of patients in each variable who have died.

†Adjusted HR is adjusted for all other variables in the table.

$\neq \mathrm{p}$ for trend.

$\S$ Result of a likelihood ratio test. 
Table 3 Demographic features of patients with non-small cell lung cancer (NSCLC) based on where they were first seen (total number with $\mathrm{NSCLC}=34$ 513)

\begin{tabular}{|c|c|c|}
\hline & $\begin{array}{l}\text { Thoracic } \\
\text { surgical centre } \\
\text { N (\%) }\end{array}$ & $\begin{array}{l}\text { Non-surgical } \\
\text { centre } \\
\text { N (\%) }\end{array}$ \\
\hline Total & 9265 (27) & $25248(73)$ \\
\hline \multicolumn{3}{|l|}{ Sex } \\
\hline Male & $5648(61)$ & $15297(61)$ \\
\hline Female & 3617 (39) & 9951 (39) \\
\hline Median (IOR) age (years) & $70(63-77)$ & $71(63-77)$ \\
\hline \multicolumn{3}{|l|}{ Performance status } \\
\hline 0 & 1804 (19) & $4043(16)$ \\
\hline 1 & 2487 (27) & 6795 (27) \\
\hline 2 & $1388(15)$ & 3929 (16) \\
\hline 3 & 824 (9) & $2427(10)$ \\
\hline 4 & $169(2)$ & $591(2)$ \\
\hline Missing & $2593(28)$ & $7463(30)$ \\
\hline \multicolumn{3}{|l|}{ Stage } \\
\hline IA & $372(4)$ & $806(3)$ \\
\hline IB & $582(6)$ & $1556(6)$ \\
\hline$\| A$ & $62(0.7)$ & $140(0.5)$ \\
\hline IIB & $381(4)$ & $1067(4)$ \\
\hline IIIA & $746(8)$ & 2031 (8) \\
\hline IIIB & $1388(15)$ & $4039(16)$ \\
\hline IV & $3003(32)$ & 7965 (32) \\
\hline Occult & 16 & 34 \\
\hline Missing & $2715(29)$ & $7610(30)$ \\
\hline \multicolumn{3}{|l|}{ Charlson Index } \\
\hline 0 & $4221(46)$ & $11352(45)$ \\
\hline 1 & 1784 (19) & $5167(20)$ \\
\hline 2 or 3 & $1564(17)$ & $4262(17)$ \\
\hline $4+$ & $1696(18)$ & $4465(18)$ \\
\hline \multicolumn{3}{|l|}{ Townsend quintile } \\
\hline 1 (most affluent) & $1116(12)$ & $4068(16)$ \\
\hline 2 & $1602(17)$ & 4791 (19) \\
\hline 3 & $1470(16)$ & $5190(21)$ \\
\hline 4 & $1724(19)$ & $5424(21)$ \\
\hline 5 (least affluent) & $3339(36)$ & $5712(23)$ \\
\hline \multicolumn{3}{|l|}{ Surgery } \\
\hline No & $7727(83)$ & $22301(88)$ \\
\hline Yes & 1538 (17) & 2947 (12) \\
\hline
\end{tabular}

The main strengths of this study are the large size and the quality of the NLCA dataset ${ }^{10}$ and the addition of an independent comorbidity score in the form of the Charlson Index. The Charlson Index was originally developed and used prospectively in a cohort of people with breast cancer, ${ }^{9}$ and it has subsequently been validated in patient cohorts encompassing both malignant ${ }^{1819}$ and non-malignant disease processes. ${ }^{20}$ Previous research has shown that the Charlson comorbidity Index is associated with lung cancer incidence ${ }^{21}$ and also survival. ${ }^{22}$

Our study found that patients with NSCLC who are first seen in a thoracic surgical centre have an advantage over those with similar disease seen at non-surgical centres with regard to access to surgery. We also found that having surgery had a large beneficial impact on survival, and this highlights the importance of access to this intervention. Previous research in this area in Scotland has shown that, as distance from a cancer centre increases, survival decreases, ${ }^{23}$ suggesting that accessibility of services is a key factor in lung cancer outcome. Other research has shown that being first seen in a radiotherapy centre is associated with an increased likelihood of receiving 'active treatment', ${ }^{24}$ and that being first seen in a specialist cancer centre is associated with a small improvement in overall survival. $^{25} 26$ There have been several large-scale reviews and policy documents in the UK designed to address inequality in cancer outcome including the Calman-Hine report, ${ }^{27}$ the NHS Cancer $\mathrm{Plan}^{28}$ and the Cancer Reform Strategy. ${ }^{29}$ Creating specialist cancer centres has been pivotal to this programme of change and, while centralising services will create greater experience and expertise in one centre, it may potentially disadvantage individuals in remote settings and increase geographical inequalities. The results of our study suggest that more reforms are needed to ensure that all people with lung cancer have equal access to surgical intervention where this is appropriate.

\section{CONCLUSIONS}

The Department of Health has published guidance that that as many as $20 \%$ of patients with NSCLC may be suitable for surgical resection. ${ }^{30}$ Our findings suggest that, if all patients with NSCLC had the same access to this intervention as those first seen at a thoracic surgical centre, the resection rates in England would increase from $13 \%$ to $17 \%$ with no detrimental impact on survival after surgery. However, what our study does not show is what aspects of 'being a surgical centre' are crucial to increasing resection rates. It is possible that this may simply be the presence of a surgeon on-site, but other aspects of the lung cancer service within these specialist centres may also be important such as the composition of the multidisciplinary team, improved access to specialist radiological and cardiovascular investigations and the geographical location of these thoracic surgical centres. Given the poor prognosis of lung cancer in the UK, understanding the care pathways in more detail and, in particular, the barriers to surgical intervention that currently exist for people seen in non-surgical centres is a pressing priority. The peer review data available at the time of this research did not influence clinical outcome measures in lung cancer, and further research is required to determine what performance measures should be collected in order to accurately describe variation in practice. As the NLCA matures, the addition of more specific information on the composition of local cancer centres and networks is essential to enable these questions to be answered.

Acknowledgements The authors acknowledge the support offered by the Biomedical Research Unit at Nottingham University, the NHS Information Centre for Health and Social Care, the Royal College of Physicians Clinical Effectiveness and Evaluation Unit (funded first author) and the British Lung Foundation (funded the second and final authors).

Funding ALR is funded by a Royal College of Physicians Clinical Quality Improvement Research and Training Fellowship. RBH is the current GSK/British Lung Foundation Professor of Respiratory Epidemiology.

\section{Competing interests None.}

Provenance and peer review Not commissioned; externally peer reviewed.

\section{REFERENCES}

1. Socinski MA, Crowell R, Hensing TE, et al. Treatment of non-small cell lung cancer, stage IV: ACCP evidence-based clinical practice guidelines (2nd edition). Chest 2007;132(3 Suppl):277-89S

2. Fry WA, Phillips JL, Menck HR. Ten-year survey of lung cancer treatment and survival in hospitals in the United States: a national cancer data base report. Cancer 1999;86:1867-76.

3. Chansky K, Sculier JP, Crowley JJ, et al. The International Association for the Study of Lung Cancer Staging Project: prognostic factors and pathologic TNM stage in surgically managed non-small cell lung cancer. J Thorac Oncol 2009;4:792-801.

4. National Institute for Clinical Excellence (NICE). The Diagnosis and Treatment of Lung Cancer. London: NICE, 2005.

5. Crook A, Duffy A, Girling DJ, et al. Survey on the treatment of non-small cell lung cancer (NSCLC) in England and Wales. Eur Respir J 1997;10:1552-8.

6. Ries LA, Eisner MP, Kosary CL, et al. SEER Cancer Statistics Review, 1975-2002. National Cancer Institute (USA), 2005.

7. Department of Health. Cancer Reform Strategy; Achieving Local Implementation-Second Annual Report. London: Department of Health, 2009 
8. Damhuis RA, Schutte PR. Resection rates and postoperative mortality in 7,899 patients with lung cancer. Eur Respir J 1996;9:7-10.

9. Charlson ME, Pompei P, Ales KL, et al. A new method of classifying prognostic comorbidity in longitudinal studies: development and validation. J Chronic Dis 1987; 40:373-83.

10. Rich AL, Tata LJ, Stanley RA, et al. Lung cancer in England: information from the National Lung Cancer Audit (LUCADA). Lung Cancer 2011;72:16-22.

11. National Lung Cancer Audit. Annual Report 2008. The NHS Information Centre for Health and Social Care, 2009. http://www.ic.nhs.uk/webfiles/Services/NCASP/audits \%20and\%20reports/NHS\%20IC\%20Lung\%20Cancer\%20AUDIT\%202009\%20FINAL. pdf.

12. National Lung Cancer Audit. Key Findings about the Quality of Care for People with Lung Cancer in England and Wales Incorporating Headline and Completeness Data from Scotland. Report for the Audit Period 2007. The NHS Information Centre for Health and Social Care, 2008. http://www.ic.nhs.uk/webfiles/Services/NCASP/audits \%20and\%20reports/7089_Lung_Cancer_V5.pdf.

13. National Lung Cancer Audit. Annual Report 2006. The NHS Information Centre for Health and Social Care, 2007. http://www.ic.nhs.uk/webfiles/Services/NCASP/audits \%20and\%20reports/19100507\%20IC\%20Lung\%20Cancer\%20Audit\%20Report\% 202006-FV.pdf.

14. Khan NF, Perera R, Harper S, et al. Adaptation and validation of the Charlson Index for Read/OXMIS coded databases. BMC Fam Pract 2010;11:1

15. Wang CY, Lin YS, Tzao C, et al. Comparison of Charlson comorbidity index and Kaplan-Feinstein index in patients with stage I lung cancer after surgical resection. Eur J Cardiothorac Surg 2007;32:877-81.

16. Asmis TR, Ding K, Seymour $L$, et al. Age and comorbidity as independent prognostic factors in the treatment of non small-cell lung cancer: a review of National Cancer Institute of Canada Clinical Trials Group trials. J Clin Oncol 2008;26:54-9.

17. National Cancer Institute. National Lung Screening Trial Results: Fast Facts. 2011. http://www.cancer.gov/newscenter/pressreleases/2011/NLSTFastFacts.
18. Cronin-Fenton DP, Norgaard $M$, Jacobsen J, et al. Comorbidity and survival of Danish breast cancer patients from 1995 to 2005. Br J Cancer 2007;96:1462-8.

19. Wildes TM, Augustin KM, Sempek D, et al. Comorbidities, not age, impact outcomes in autologous stem cell transplant for relapsed non-Hodgkin lymphoma. Biol Blood Marrow Transplant 2008;14:840-6.

20. Charlson M, Szatrowski TP, Peterson J, et al. Validation of a combined comorbidity index. J Clin Epidemiol 1994;47:1245-51.

21. Dalton S0, Steding-Jessen M, Engholm G, et al. Social inequality and incidence of and survival from lung cancer in a population-based study in Denmark, 1994-2003. Eur J Cancer 2008;44:1989-95.

22. Birim 0, Kappetein AP, Bogers AJ. Charlson comorbidity index as a predictor of long-term outcome after surgery for nonsmall cell lung cancer. Eur J Cardiothorac Surg 2005;28:759-62.

23. Campbell NC, Elliott AM, Sharp $L$, et al. Rural factors and survival from cancer: analysis of Scottish cancer registrations. Br J Cancer 2000:82:1863-6.

24. Jack RH, Gulliford MC, Ferguson J, et al. Geographical inequalities in lung cancer management and survival in South East England: evidence of variation in access to oncology services? Br J Cancer 2003;88:1025-31.

25. Jones AP, Haynes R, Sauerzapf V, et al. Travel times to health care and survival from cancers in Northern England. Eur J Cancer 2008:44:269-74.

26. Stiller CA. Centralised treatment, entry to trials and survival. $\mathrm{Br} \mathrm{J}$ Cancer 1994;70:352-62

27. Department of Health. A Policy Framework for Commissioning Cancer Services: a Report by the Expert Advisory Group on Cancer to the Chief Medical Officers of England and Wales. London: Department of Health, 1995.

28. Department of Health. The NHS Cancer Plan. London: Department of Health, 2000.

29. Department of Health. Cancer Reform Strategy. London: Department of Health 2007.

30. Department of Health. Guidance on Commissioning Cancer Services; Improving Outcomes in Lung Cancer: The Manual.London: Department of Health, 1998.

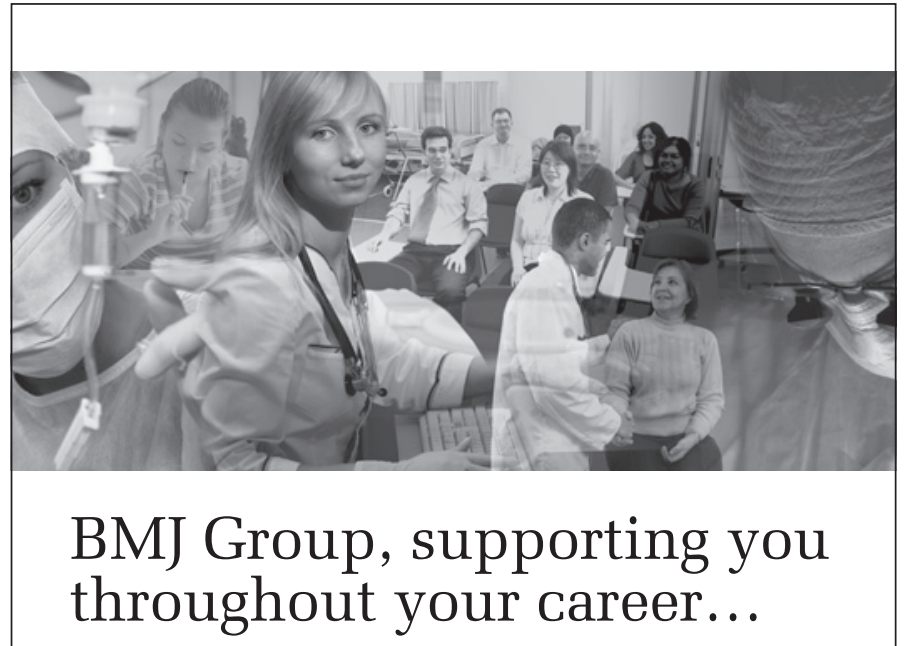

\begin{abstract}
At BMJ Group we have resources available to you at every stage of your career.

Whether you are a medical student or doctor in training looking to keep up with the latest news and prepare for exams, or a qualified doctor who wants the latest medical information, to attend conferences, or looking for your next job, BMJ Group has something to offer. For the latest information on all of our products and services register to receive email updates at
\end{abstract}

group.bmj.com/registration 